\title{
Oscillation criteria for third-order delay differential equations
}

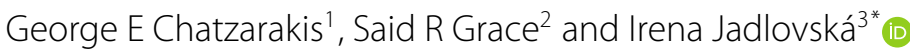

\author{
"Correspondence: \\ irena.jadlovska@tuke.sk \\ ${ }^{3}$ Department of Mathematics and \\ Theoretical Informatics, Faculty of \\ Electrical Engineering and \\ Informatics, Technical University of \\ Košice, Letná 9, Košice, 042 00, \\ Slovakia \\ Full list of author information is \\ available at the end of the article
}

\begin{abstract}
The objective in this paper is to study the oscillatory and asymptotic behavior of the solutions of a linear third-order delay differential equation of the form

$$
\left(r_{2}(t)\left(r_{1}(t) y^{\prime}(t)\right)^{\prime}\right)^{\prime}+q(t) y(\tau(t))=0
$$

We establish new oscillation criteria that can be used to test for oscillations, even when the previously known criteria fail to apply. Examples illustrating the results are also given.
\end{abstract}

MSC: $34 \mathrm{C} 10 ; 34 \mathrm{~K} 11$

Keywords: third-order; differential equation; delay; Grönwall inequality; oscillation

\section{Introduction}

In this work, we are concerned with the oscillation of a linear third-order delay differential equation of the form

$$
\left(r_{2}(t)\left(r_{1}(t) y^{\prime}(t)\right)^{\prime}\right)^{\prime}+q(t) y(\tau(t))=0, \quad t \in I:=\left[t_{0}, \infty\right),
$$

where $t_{0}>0$ is a fixed real number, $r_{1}, r_{2}, q \in \mathcal{C}(I, \mathbb{R})$ are positive functions and the delay function $\tau \in \mathcal{C}^{1}(I, \mathbb{R})$ satisfies $\tau(t)<t, \tau^{\prime}(t) \geq 0$ and $\tau(t) \rightarrow \infty$ as $t \rightarrow \infty$.

Throughout, we will always assume that

$$
\int_{t_{0}}^{\infty} \frac{1}{r_{1}(s)} \mathrm{d} s=\int_{t_{0}}^{\infty} \frac{1}{r_{2}(s)} \mathrm{d} s=\infty
$$

By a solution of (1.1) we mean a function $y(t) \in \mathcal{C}^{1}\left[t_{y}, \infty\right)$ which has the property $r_{1}(t) y^{\prime}(t) \in$ $\mathcal{C}^{1}\left[t_{y}, \infty\right)$ and $r_{2}(t)\left(r_{1}(t) y^{\prime}(t)\right)^{\prime} \in \mathcal{C}^{1}\left[t_{y}, \infty\right)$ and satisfies (1.1) on $\left[t_{y}, \infty\right)$ for every $t \geq t_{y} \geq t_{0}$.

We restrict our attention to those solutions of (1.1) which exist on $I$ and satisfy the condition $\sup \left\{|x(t)|: t \geq t_{1}\right\}>0$ for any $t_{1} \geq t_{y}$. We assume that (1.1) possesses such a solution.

A solution $y(t)$ of (1.1) is said to be oscillatory if it is neither eventually positive nor eventually negative. Otherwise, it is said to be nonoscillatory. The equation itself is termed oscillatory if all of its solutions oscillate.

As is well known, differential equations of third-order have long been considered as valuable tools in the modeling of many phenomena in different areas of applied mathematics

(c) The Author(s) 2017. This article is distributed under the terms of the Creative Commons Attribution 4.0 International License (http://creativecommons.org/licenses/by/4.0/), which permits unrestricted use, distribution, and reproduction in any medium, provided you give appropriate credit to the original author(s) and the source, provide a link to the Creative Commons license, and indicate if changes were made. 
and physics. Indeed, it is worthwhile to mention their use in the study of entry-flow phenomenon [1], the propagation of electrical pulses in the nerve of a squid approximated by the famous Nagumo's equation [2], the feedback nuclear reactor problem [3] and so on.

Hence, a great amount of work has been done over the last three decades on the oscillation theory of third-order differential equations with variable coefficients. The most significant results published up to 2014 have been collected and summarized in recent monographs $[4,5]$.

It follows from the generalized Kiguradze lemma (cf. Lemma 1) that the first derivative of any positive solution $y$ of (1.1) is of one sign eventually, i.e., $y$ is either increasing or decreasing. Since in the ordinary case (when $\tau(t) \equiv t$ ) there always exists a decreasing solution of (1.1), see [6, Lemma 1], authors have used various techniques to present sufficient conditions guaranteeing that any solution of (1.1) oscillates or converges to zero eventually. For such results, we refer the reader to [6-14] and the references cited therein.

However, it is interesting to note that the delay argument can cause that (1.1) becomes oscillatory. As an example of this property, we can consider the third-order differential equation $y^{\prime \prime \prime}(t)+y(t-\tau)=0, \tau>0$, which is oscillatory if and only if $\tau$ e $>3$ (see [15, Theorem 1]). But the corresponding third-order ordinary differential equation $y^{\prime \prime \prime}(t)+y(t)=0$ has a nonoscillatory solution $y(t)=\mathrm{e}^{-t}$. Therefore, it is of special interest to establish new criteria ensuring oscillation of all solutions of $(1.1)$ when $\tau(t)<t$.

An interesting method in oscillation theory is to use some comparison principles based on which the oscillatory behavior of the solutions of the studied differential equation is inherited from the oscillations in a first-order delay differential equations, resulting in conditions involving 1/e. The results concerned with this problem for (1.1) and its various generalizations were presented in $[7,16-22]$.

For a particular case of (1.1), namely,

$$
\left(r_{2}(t) y^{\prime \prime}(t)\right)^{\prime}+q(t) y(\tau(t))=0
$$

Baculíková and Džurina [17] established the following results.

Theorem A (See [17, Theorem 2]) Assume that

$$
\int_{t_{0}}^{\infty} \int_{v}^{\infty} \frac{1}{r_{2}(u)} \int_{u}^{\infty} q(s) \mathrm{d} s \mathrm{~d} u \mathrm{~d} v=\infty
$$

If the first-order delay differential equation

$$
x^{\prime}(t)+\left(q(t) \int_{t_{0}}^{\tau(t)} \frac{\tau(t)-u}{r_{2}(u)} \mathrm{d} u\right) x(\tau(t))=0
$$

is oscillatory, then every solution of (1.2) is either oscillatory or converges to zero as $t \rightarrow \infty$.

Theorem B (See [17, Theorem 3]) Let there exist a function $\xi(t) \in \mathcal{C}^{1}(I, \mathbb{R})$ such that

$$
\xi^{\prime}(t) \geq 0, \quad \xi(t)>t, \quad \eta(t)=\tau(\xi(\xi(t)))<t .
$$


If both first-order delay differential equations (1.4) and

$$
z^{\prime}(t)+\left(\int_{t}^{\xi(t)} \frac{1}{r_{2}(v)} \int_{v}^{\xi(v)} q(u) \mathrm{d} u \mathrm{~d} v\right) z(\eta(t))=0
$$

are oscillatory, then (1.2) is oscillatory.

It is useful to note that oscillations in (1.4) rule out increasing positive solutions of (1.1). The proof of Theorem A is essentially based on using the following estimate relating an increasing positive solution of (1.2) with its second quasi-derivative:

$$
y(\tau(t)) \geq r_{2}(\tau(t)) y^{\prime \prime}(\tau(t)) \int_{t_{1}}^{\tau(t)} \frac{\tau(t)-u}{r_{2}(u)} \mathrm{d} u .
$$

A similar estimate for increasing positive solutions of (1.1), namely,

$$
y(\tau(t)) \geq r_{2}(\tau(t))\left(r_{1}(\tau(t)) y^{\prime}(\tau(t))\right)^{\prime} \int_{t_{1}}^{\tau(t)} \frac{1}{r_{1}(s)} \int_{t_{1}}^{s} \frac{1}{r_{2}(u)} \mathrm{d} u \mathrm{~d} s
$$

was given in [7, Lemma 4]. In fact, inequality (1.6) in a more or less general form was repeatedly used in the process of elimination of increasing positive solutions in (1.1), its generalizations or particular cases; see, e.g., [7, 21-26]. It is clear after checking that, despite the differences in the proofs of the cited works, the resulting criteria have in common that their strength depends on the sharpness of estimate (1.6).

On the other hand, oscillations in (1.5) eliminate the existence of positive decreasing solutions in (1.2). Since there is no general rule as to how to choose a function $\xi(t)$ satisfying the imposed conditions, an interesting problem is how to establish a corresponding result without requiring the existence of the unknown function $\xi(t)$. Here, we will also address this problem.

The main objective in this paper is to study the asymptotic and oscillatory behavior of the solutions of (1.1). Our method is essentially based on establishing sharper estimates for increasing positive solutions of (1.1) than (1.6), using an iterative technique; and to obtain analogous iterative estimates for decreasing positive solutions of (1.1). Similar ideas as those presented here have been successively employed in investigating oscillatory behavior of second-order advanced differential equations, see a recent work [27] for details. The results obtained are new even in the case of (1.1) when $r_{i}(t)=1, i=1,2$. Furthermore, the iterative nature of the results enables us to test for oscillations, even when the previously known results fail to apply. We demonstrate the improvement we achieve with these results by applying them to Euler-type delay differential equations.

Remark 1 In the sequel, all functional inequalities are assumed to hold eventually, that is, they are satisfied for all $t$ large enough.

Remark 2 Without loss of generality, we can deal only with the positive solutions of (1.1).

\section{Some lemmas and auxiliary results}

In this section, we state and prove some lemmas that will be useful in establishing our main results. For completeness, we start by recalling the adaptation of the generalized Kiguradze 
lemma. Next, we will provide some important monotonic properties of a positive solution $y$ of (1.1) in cases (2.1) and (2.2), respectively.

For the sake of brevity, we define

$$
L_{0} y(t)=y(t), \quad L_{i} y(t)=r_{i}(t)\left(L_{i-1} y(t)\right)^{\prime}, \quad i=1,2, \quad L_{3} y(t)=\left(L_{2} y(t)\right)^{\prime},
$$

for $t \in I$. Using this notation, equation (1.1) takes the form

$$
L_{3} y(t)+q(t) y(\tau(t))=0 .
$$

Lemma 1 (See [28, Lemma 2]) Assume that $y(t)$ is an eventually positive solution of (1.1). Then $y(t)$ satisfies one of the following two cases:

$$
\begin{array}{llll}
y(t)>0, & L_{1} y(t)>0, & L_{2} y(t)>0, & L_{3} y(t)<0, \\
y(t)>0, & L_{1} y(t)<0, & L_{2} y(t)>0, & L_{3} y(t)<0,
\end{array}
$$

eventually.

Lemma 2 Assume that $y(t)$ is a positive solution of (1.1) which satisfies (2.1). Denote

$$
\begin{aligned}
& R_{1}\left(t, t_{*}\right):=\int_{t_{*}}^{t} \frac{1}{r_{1}(v)} \int_{t_{*}}^{v} \frac{1}{r_{2}(s)} \mathrm{d} s \mathrm{~d} v, \\
& R_{m+1}\left(t, t_{*}\right):=\int_{t_{*}}^{t} \frac{1}{r_{1}(v)} \int_{t_{*}}^{v} \frac{1}{r_{2}(s)} \exp \left(\int_{s}^{t} q(u) R_{m}\left(\tau(u), t_{*}\right) \mathrm{d} u\right) \mathrm{d} s \mathrm{~d} v, \quad m \in \mathbb{N},
\end{aligned}
$$

for $t \geq t_{*}$, where $t_{*} \in I$ is large enough. Then

$$
y(\tau(t)) \geq L_{2} y(\tau(t)) R_{m}\left(\tau(t), t_{*}\right), \quad t \geq t_{*} .
$$

Proof Let $y(t)$ be a solution of (1.1) satisfying $(2.1)$ on $\left[t_{1}, \infty\right), t_{1} \in I$. Since $L_{2} y(t)$ is nonincreasing, it is easy to see that

$$
L_{1} y(t) \geq \int_{t_{1}}^{t}\left(L_{1} y(s)\right)^{\prime} \mathrm{d} s=\int_{t_{1}}^{t} \frac{1}{r_{2}(s)} L_{2} y(s) \mathrm{d} s \geq L_{2} y(t) \int_{t_{1}}^{t} \frac{1}{r_{2}(s)} \mathrm{d} s
$$

or

$$
y^{\prime}(t) \geq L_{2} y(t) \frac{1}{r_{1}(t)} \int_{t_{1}}^{t} \frac{1}{r_{2}(s)} \mathrm{d} s, \quad t \geq t_{1} .
$$

Integrating the above inequality from $t_{1}$ to $t$ and using (2.3), we have

$$
y(t) \geq \int_{t_{1}}^{t} L_{2} y(v) \frac{1}{r_{1}(v)} \int_{t_{1}}^{v} \frac{1}{r_{2}(s)} \mathrm{d} s \mathrm{~d} v \geq L_{2} y(t) R_{1}\left(t, t_{1}\right) .
$$

Obviously, there exists $t_{2} \geq t_{1}$ such that for all $t \geq t_{2}$,

$$
y(\tau(t)) \geq L_{2} y(\tau(t)) R_{1}\left(\tau(t), t_{2}\right) .
$$

That is, (2.4) is satisfied for $m=1$. 
Now, proceeding to the next induction step, we assume that (2.4) holds for some $m>1$, which means that

$$
y(\tau(t)) \geq L_{2} y(\tau(t)) R_{m}\left(\tau(t), t_{*}\right), \quad t \geq t_{*} \geq t_{2}
$$

Combining (2.5) and (1.1), we get

$$
L_{3} y(t)+q(t) R_{m}\left(\tau(t), t_{*}\right) L_{2} y(\tau(t)) \leq 0
$$

or

$$
x^{\prime}(t)+q(t) R_{m}\left(\tau(t), t_{*}\right) x(\tau(t)) \leq 0,
$$

where $x(t):=L_{2} y(t)$. In view of $x(\tau(t)) \geq x(t)$, we can write the last inequality in the form

$$
x^{\prime}(t)+q(t) R_{m}\left(\tau(t), t_{*}\right) x(t) \leq 0 .
$$

Applying the Grönwall inequality in (2.7), we obtain

$$
x(s) \geq x(t) \exp \left(\int_{s}^{t} q(u) R_{m}\left(\tau(u), t_{*}\right) \mathrm{d} u\right), \quad t \geq s \geq t_{*}
$$

or,

$$
L_{2} y(s) \geq L_{2} y(t) \exp \left(\int_{s}^{t} q(u) R_{m}\left(\tau(u), t_{*}\right) \mathrm{d} u\right), \quad t \geq s \geq t_{*} .
$$

Therefore,

$$
L_{1} y(t) \geq \int_{t_{*}}^{t} \frac{1}{r_{2}(s)} L_{2} y(s) \mathrm{d} s \geq L_{2} y(t) \int_{t_{*}}^{t} \frac{1}{r_{2}(s)} \exp \left(\int_{s}^{t} q(u) R_{m}\left(\tau(u), t_{*}\right) \mathrm{d} u\right) \mathrm{d} s,
$$

that is,

$$
y^{\prime}(t) \geq L_{2} y(t) \frac{1}{r_{1}(t)} \int_{t_{*}}^{t} \frac{1}{r_{2}(s)} \exp \left(\int_{s}^{t} q(u) R_{m}\left(\tau(u), t_{*}\right) \mathrm{d} u\right) \mathrm{d} s
$$

Integrating the above inequality from $t_{*}$ to $t$ and using (2.8) again, we obtain

$$
\begin{aligned}
y(t) \geq & \int_{t_{*}}^{t} L_{2} y(v) \frac{1}{r_{1}(v)} \int_{t_{*}}^{v} \frac{1}{r_{2}(s)} \exp \left(\int_{s}^{v} q(u) R_{m}\left(\tau(u), t_{*}\right) \mathrm{d} u\right) \mathrm{d} s \mathrm{~d} v \\
\geq & L_{2} y(t) \int_{t_{*}}^{t} \exp \left(\int_{v}^{t} q(u) R_{m}\left(\tau(u), t_{*}\right) \mathrm{d} u\right) \\
& \times \frac{1}{r_{1}(v)} \int_{t_{*}}^{v} \frac{1}{r_{2}(s)} \exp \left(\int_{s}^{v} q(u) R_{m}\left(\tau(u), t_{*}\right) \mathrm{d} u\right) \mathrm{d} s \mathrm{~d} v \\
= & L_{2} y(t) \int_{t_{*}}^{t} \frac{1}{r_{1}(v)} \int_{t_{*}}^{v} \frac{1}{r_{2}(s)} \exp \left(\int_{s}^{t} q(u) R_{m}\left(\tau(u), t_{*}\right) \mathrm{d} u\right) \mathrm{d} s \mathrm{~d} v .
\end{aligned}
$$


Hence, there exists $t_{* *} \geq t_{*}$ such that for all $t \geq t_{* *}$,

$$
y(\tau(t)) \geq L_{2} y(\tau(t)) \int_{t_{* *}}^{\tau(t)} \frac{1}{r_{1}(v)} \int_{t_{* *}}^{v} \frac{1}{r_{2}(s)} \exp \left(\int_{s}^{\tau(t)} q(u) R_{m}\left(\tau(u), t_{* *}\right) \mathrm{d} u\right) \mathrm{d} s \mathrm{~d} v
$$

which, in view of (2.3), becomes

$$
y(\tau(t)) \geq L_{2} y(\tau(t)) R_{m+1}\left(\tau(t), t_{* *}\right), \quad t \geq t_{* *} .
$$

This completes the induction step and the proof of the lemma.

Remark 3 Note that, for $m=1$, (2.4) reduces to (1.6).

Lemma 3 Assume that $y(t)$ is an eventually positive solution of (1.1) which satisfies (2.2). Denote

$$
\begin{aligned}
& \tilde{R}_{1}(v, u):=\int_{u}^{v} \frac{1}{r_{1}(x)} \int_{x}^{v} \frac{1}{r_{2}(s)} \mathrm{d} s \mathrm{~d} x \\
& \tilde{R}_{n+1}(v, u):=\int_{u}^{v} \frac{1}{r_{1}(x)} \int_{x}^{v} \frac{1}{r_{2}(s)} \exp \left(\int_{s}^{v} q(z) R_{n}(z, \tau(z)) \mathrm{d} z\right) \mathrm{d} s \mathrm{~d} x, \quad n \in \mathbb{N},
\end{aligned}
$$

for $v \geq u \geq t_{*}$, where $t_{*} \in I$ is large enough. Then

$$
y(u) \geq L_{2} y(v) \tilde{R}_{n}(v, u), \quad v \geq u \geq t_{*}
$$

Proof Let $y(t)$ be a solution of (1.1) satisfying (2.2) on $\left[t_{1}, \infty\right), t_{1} \in I$. Since $L_{2} y(t)$ is nonincreasing, we may write

$$
-L_{1} y(u) \geq L_{1} y(v)-L_{1} y(u)=\int_{u}^{v} \frac{1}{r_{2}(s)} L_{2} y(s) \mathrm{d} s \geq L_{2} y(v) \int_{u}^{v} \frac{1}{r_{2}(s)} \mathrm{d} s
$$

for some $v \geq u \geq t_{1}$, and hence,

$$
-y^{\prime}(u) \geq L_{2} y(v) \frac{1}{r_{1}(u)} \int_{u}^{v} \frac{1}{r_{2}(s)} \mathrm{d} s .
$$

Integrating the above inequality from $u$ to $v \geq u \geq t_{1}$, we get

$$
y(u) \geq L_{2} y(v) \int_{u}^{v} \frac{1}{r_{1}(x)} \int_{x}^{v} \frac{1}{r_{2}(s)} \mathrm{d} s \mathrm{~d} x=L_{2} y(v) \tilde{R}_{1}(v, u),
$$

which means that (2.11) is satisfied for $n=1$.

Now, assume that (2.11) holds for some $n>1$, i.e.,

$$
y(u) \geq L_{2} y(v) \tilde{R}_{n}(v, u), \quad v \geq u \geq t_{*} \geq t_{1} .
$$

Clearly, using (2.14) with $u=\tau(t)$ and $v=t$ in (1.1) yields

$$
L_{3} y(t)+q(t) \tilde{R}_{n}(t, \tau(t)) L_{2} y(t) \leq 0 .
$$


Proceeding as in the proof of Lemma 2, we get

$$
L_{2} y(s) \geq L_{2} y(v) \exp \left(\int_{s}^{v} q(z) \tilde{R}_{n}(z, \tau(z)) \mathrm{d} z\right), \quad v \geq s \geq t_{*} .
$$

Using (2.15) in (2.12), we have

$$
-L_{1} y(u) \geq L_{2} y(v) \int_{u}^{v} \frac{1}{r_{2}(s)} \exp \left(\int_{s}^{v} q(z) \tilde{R}_{n}(z, \tau(z)) \mathrm{d} z\right) \mathrm{d} s,
$$

and therefore,

$$
-y^{\prime}(u) \geq L_{2} y(v) \frac{1}{r_{1}(u)} \int_{u}^{v} \frac{1}{r_{2}(s)} \exp \left(\int_{s}^{v} q(z) \tilde{R}_{n}(z, \tau(z)) \mathrm{d} z\right) \mathrm{d} s .
$$

Finally, by integrating (2.16) from $u$ to $v \geq u \geq t_{*}$, we conclude that

$$
\begin{aligned}
y(u) & \geq L_{2} y(v) \int_{u}^{v} \frac{1}{r_{1}(x)} \int_{x}^{v} \frac{1}{r_{2}(s)} \exp \left(\int_{s}^{v} q(z) \tilde{R}_{n}(z, \tau(z)) \mathrm{d} z\right) \mathrm{d} s \mathrm{~d} x \\
& =L_{2} y(v) \tilde{R}_{n+1}(v, u) .
\end{aligned}
$$

Thus, relation (2.11) holds for any $n \in \mathbb{N}$. The proof is complete.

Lemma 4 Assume that $y(t)$ is an eventually positive solution of (1.1) which satisfies (2.2).

If

$$
\int_{t_{0}}^{\infty} \frac{1}{r_{1}(v)} \int_{v}^{\infty} \frac{1}{r_{2}(u)} \int_{u}^{\infty} q(s) \mathrm{d} s \mathrm{~d} u \mathrm{~d} v=\infty
$$

then $y(t)$ tends to zero as $t \rightarrow \infty$.

Proof Since the proof is similar to that of [17, Lemma 2], we omit it.

\section{Main results}

We are prepared to provide the main results of the paper. At first, we improve Theorem A by establishing a new sufficient condition for all nonoscillatory solutions of (1.1) to converge to zero as $t \rightarrow \infty$. Second, we relax condition (2.17) and employ another one in order to eliminate (2.2)-type solutions in (1.1) and attain oscillation of (1.1).

Theorem 1 Let (2.17) hold and $R_{m}\left(t, t_{*}\right)$ be defined by (2.3). If the first-order delay differential equation

$$
x^{\prime}(t)+q(t) R_{m}\left(\tau(t), t_{*}\right) x(\tau(t))=0
$$

is oscillatory for some $m \in \mathbb{N}$ and $t_{*} \in I$, then every solution of (1.1) is either oscillatory or tends to zero as $t \rightarrow \infty$.

Proof Let $y(t)$ be a nonoscillatory solution of (1.1), say $y(t)>0$ and $y(\tau(t))>0$ for $t \geq T_{1}$ for some $T_{1} \geq t_{0}$. By Lemma 1, $y(t)$ satisfies either (2.1) or (2.2) for $t \geq t_{1} \geq T_{1}$. 
Assume first that $y(t)$ satisfies (2.1) for $t \geq t_{1}$. Combining (2.4) with (1.1), we see that $x(t):=L_{2} y(t)$ is a positive solution of the first-order delay differential inequality

$$
x^{\prime}(t)+q(t) R_{m}\left(\tau(t), t_{*}\right) x(\tau(t)) \leq 0 .
$$

Then, by virtue of [29, Theorem 1], the associated delay differential equation (3.1) also has a positive solution, which is a contradiction. The proof is complete.

Now, assume that $y(t)$ satisfies (2.2) for $t \geq t_{1}$. By Lemma 4 , we have $\lim _{t \rightarrow \infty} y(t)=0$. The proof is complete.

Applying the known oscillation criteria for (3.1), one immediately gets an oscillation criterion for (1.1). The following is due to Ladde et al. [30, Theorem 2.1.1].

Corollary 1 Let (2.17) hold and $R_{m}\left(t, t_{*}\right)$ be defined by (2.3). If

$$
\liminf _{t \rightarrow \infty} \int_{\tau(t)}^{t} q(s) R_{m}\left(\tau(s), t_{*}\right) \mathrm{d} s>\frac{1}{\mathrm{e}}
$$

for some $m \in \mathbb{N}$ and $t_{*} \in I$, then every solution of (1.1) is either oscillatory or tends to zero as $t \rightarrow \infty$.

Theorem 2 Let $R_{m}\left(t, t_{*}\right)$ and $\tilde{R}_{n}(v, u)$ be defined by (2.3) and (2.10), respectively. If the firstorder delay differential equation (3.1) is oscillatory for some $m \in \mathbb{N}$ and $t_{*} \in \mathcal{I}$, and

$$
\limsup _{t \rightarrow \infty} \int_{\tau(t)}^{t} q(s) \tilde{R}_{n}(\tau(t), \tau(s)) \mathrm{d} s>1,
$$

for some $n \in \mathbb{N}$, then (1.1) is oscillatory.

Proof Let $y(t)$ be a nonoscillatory solution of (1.1), say $y(t)>0$ and $y(\tau(t))>0$ for $t \geq T_{1}$ for some $T_{1} \geq t_{0}$. By Lemma $1, y(t)$ satisfies either (2.1) or (2.2) for $t \geq t_{1} \geq T_{1}$.

Assume first that $y(t)$ satisfies (2.1) for $t \geq t_{1}$. Proceeding as in the proof of Theorem 1 , we arrive at a contradiction.

Now, assume that $y(t)$ satisfies (2.2) for $t \geq t_{1}$. Integrating (1.1) from $\tau(t)$ to $t$, we have

$$
L_{2} y(\tau(t)) \geq L_{2} y(\tau(t))-L_{2} y(t)=\int_{\tau(t)}^{t} q(s) y(\tau(s)) \mathrm{d} s
$$

Using (2.11) in (3.4) with $u=\tau(s)$ and $v=\tau(t)$, we see that

$$
L_{2} y(\tau(t)) \geq L_{2} y(\tau(t)) \int_{\tau(t)}^{t} q(s) \tilde{R}_{n}(\tau(t), \tau(s)) \mathrm{d} s .
$$

Taking the limsup as $t \rightarrow \infty$ on both sides of the above inequality, we are led to a contradiction with (3.3). The proof is complete.

\section{Examples}

The following examples are provided to show the improvement we achieve by our results over those presented earlier. All numerical calculations can be easily performed in MATLAB. 
Example 1 Consider the third-order differential equation of the Euler type:

$$
y^{\prime \prime \prime}(t)+\frac{3}{t^{3}} y(0.5 t)=0, \quad t \geq 1
$$

For $m=1$, the left-hand side of condition (3.1) gives

$$
0.25993<1 / \text { e. }
$$

However, for $m=2$, we get

$$
0.511863>1 / \mathrm{e}
$$

that is, (3.1) is satisfied for $m=2$. On the other hand, it is easy to verify that (2.17) holds. Thus, from Corollary 1 , we conclude that every solution of (4.1) is either oscillatory or tends to zero as $t \rightarrow \infty$. One such nonoscillatory solution is $y(t)=t^{-1}$.

We remark that Theorem A, as well as [7, Theorem 1], [22, Theorem 12], [25, Theorem 2.9], fail due to (4.2).

Example 2 Consider the third-order differential equation of the Euler type

$$
y^{\prime \prime \prime}(t)+\frac{18}{t^{3}} y(0.5 t)=0, \quad t \geq 1
$$

From Example 1, we know that (3.1) is satisfied for $m=1$. On the other hand, the left-hand side of (3.3) gives

$$
\begin{aligned}
& 0.643702<1, \\
& 0.874346<1,
\end{aligned}
$$

and

$$
1.364297>1
$$

for $n=1,2,3$, respectively. Thus, (3.3) is satisfied for $n=3$, and from Theorem 2 we conclude that (4.3) is oscillatory. We stress that, unlike the results in $[9,10,16-20,25,31]$, we do not require the existence of a suitable auxiliary function.

\section{Summary}

In this paper, we have obtained new oscillation criteria for (1.1), which, to the best of our knowledge, essentially improve a number of related results reported in the literature, even in the case when $r_{1}=r_{2} \equiv 1$.

Our approach is based on refining classical techniques, in which a desired property of the studied equation is deduced from oscillation of first-order equations by taking into account such part of the overall impact of the delay which has been neglected in earlier results.

An interesting problem for further research is to establish different iterative techniques for testing oscillations in (1.1) independently on the constant 1/e. 


\section{Acknowledgements}

We are grateful to the editors and three anonymous referees for a very careful reading of the manuscript and for pointing out several inaccuracies. The work on this research has been supported by the internal grant project No. FEl-2015-22.

Funding

Not applicable.

Availability of data and materials

Not applicable.

Ethics approval and consent to participate

Not applicable.

\section{Competing interests}

The authors declare that they have no competing interests.

\section{Consent for publication}

Not applicable.

\section{Authors' contributions}

The authors contributed equally and significantly in writing this paper. All authors read and approved the final manuscript.

\section{Author details}

${ }^{1}$ Department of Electrical and Electronic Engineering Educators, School of Pedagogical and Technological Education (ASPETE), N. Heraklio, Athens, 14121, Greece. ²Department of Engineering Mathematics, Faculty of Engineering, Cairo University, Orman, Giza, 12221, Egypt. ${ }^{3}$ Department of Mathematics and Theoretical Informatics, Faculty of Electrical Engineering and Informatics, Technical University of Košice, Letná 9, Košice, 042 00, Slovakia.

\section{Publisher's Note}

Springer Nature remains neutral with regard to jurisdictional claims in published maps and institutional affiliations.

\section{Received: 24 August 2017 Accepted: 2 October 2017 Published online: 13 October 2017}

\section{References}

1. Jayaraman, G, Padmanabhan, N, Mehrotra, R: Entry flow into a circular tube of slowly varying cross-section. Fluid Dyn. Res. 1(2), 131-144 (1986)

2. McKean, HP: Nagumo's equation. Adv. Math. 4(3), 209-223 (1970)

3. Vreeke, SA, Sandquist, GM: Phase space analysis of reactor kinetics. Nucl. Sci. Eng. 42(3), 295-305 (1970)

4. Padhi, S, Pati, S: Theory of Third-Order Differential Equations. Springer, New Delhi (2014)

5. Saker, S: Oscillation Theory of Delay Differential and Difference Equations: Second and Third Orders. LAP Lambert Academic Publishing (2010)

6. Cecchi, M, Došlá, Z, Marini, M: Disconjugate operators and related differential equations. In: Proceedings of the 6th Colloquium on the Qualitative Theory of Differential Equations, Szeged, 1999. Proc. Colloq. Qual. Theory Differ. Equ. (1999). Electron. J. Qual. Theory Differ. Equ. 6, 4 (2000)

7. Agarwal, RP, Aktas, MF, Tiryaki, A: On oscillation criteria for third order nonlinear delay differential equations. Arch. Math. 45(1), 1-18 (2009)

8. Aktaş, M, Tiryaki, A, Zafer, A: Oscillation criteria for third-order nonlinear functional differential equations. Appl. Math. Lett. 23(7), 756-762 (2010)

9. Baculíková, B, Elabbasy, EM, Saker, SH, Džurina, J: Oscillation criteria for third-order nonlinear differential equations. Math. Slovaca 58(2), 201-220 (2008)

10. Saker, SH, Džurina, J: On the oscillation of certain class of third-order nonlinear delay differential equations. Math. Bohem. 135(3), 225-237 (2010)

11. Škerlík, A: Integral criteria of oscillation for a third order linear differential equation. Math. Slovaca 45(4), 403-412 (1995)

12. Grace, SR, Agarwal, RP, Aktas, MF: On the oscillation of third order functional differential equations. Indian J. Pure Appl. Math. 39(6), 491-507 (2008)

13. Aktas, MF, Tiryaki, A, Zafer, A: Integral criteria for oscillation of third order nonlinear differential equations. Nonlinear Anal. 71(12), e1496-e1502 (2009)

14. Aktaş, MF, Tiryaki, A: Oscillation criteria for a class of third-order nonlinear delay differential equations. In: Further Progress in Analysis, pp. 507-514. World Scientific, Hackensack (2009)

15. Ladas, G, Sficas, YG, Stavroulakis, IP: Necessary and sufficient conditions for oscillations of higher order delay differential equations. In: Differential Equations, Birmingham, Ala., 1983. North-Holland Math. Stud., vol. 92, pp. 373-379. North-Holland, Amsterdam (1984)

16. Agarwal, RP, Bohner, M, Li, T, Zhang, C: Oscillation of third-order nonlinear delay differential equations. Taiwan. J. Math. 17(2), 545-558 (2013)

17. Baculíková, B, Džurina, J: Oscillation of third-order nonlinear differential equations. Appl. Math. Lett. 24(4), 466-470 (2011)

18. Baculíková, B, Džurina, J: Oscillation of third-order functional differential equations. Electron. J. Qual. Theory Differ. Equ. 43, 1 (2010) 
19. Candan, T, Dahiya, RS: Oscillation of third order functional differential equations with delay. In: Proceedings of the Fifth Mississippi State Conference on Differential Equations and Computational Simulations, Mississippi State, MS 2001. Electron. J. Differ. Equ. Conf., vol. 10, pp. 79-88. Southwest Texas State University, San Marcos (2003)

20. Elabbasy, EM, Hassan, TS, Elmatary, BM: Oscillation criteria for third order delay nonlinear differential equations. Electron. J. Qual. Theory Differ. Equ. 5, 11 (2012)

21. Bohner, M, Grace, SR, Jadlovská, I: Oscillation criteria for third-order functional differential equations with damping. Electron. J. Differ. Equ. 215, 15 (2016)

22. Baculíková, B, Džurina, J, Rogovchenko, YV: Oscillation of third order trinomial delay differential equations. Appl. Math Comput. 218(13), 7023-7033 (2012)

23. Gao, L, Zhang, Q, Liu, S: Oscillatory behavior of third-order nonlinear delay dynamic equations on time scales. J. Comput. Appl. Math. 256, 104-113 (2014)

24. Hassan, TS: Oscillation of third order nonlinear delay dynamic equations on time scales. Math. Comput. Model. 49(7-8), 1573-1586 (2009)

25. Grace, SR: Oscillation criteria for third order nonlinear delay differential equations with damping. Opusc. Math. 35(4), 485-497 (2015)

26. Li, T, Zhang, C, Baculí ková, B, Džurina, J: On the oscillation of third-order quasi-linear delay differential equations. Tatra Mt. Math. Publ. 48, 117-123 (2011)

27. Jadlovska, I: Iterative oscillation results for second-order differential equations with advanced argument. Electron. J. Differ. Equ. (2017)

28. Kusano, T, Naito, M: Comparison theorems for functional-differential equations with deviating arguments. J. Math Soc. Jpn. 33(3), 509-532 (1981)

29. Philos, CG: On the existence of nonoscillatory solutions tending to zero at $\infty$ for differential equations with positive delays. Arch. Math. (Basel) 36(2), 168-178 (1981)

30. Ladde, GS, Lakshmikantham, V, Zhang, BG: Oscillation Theory of Differential Equations with Deviating Arguments. Monographs and Textbooks in Pure and Applied Mathematics, vol. 110. Dekker, New York (1987)

31. Grace, SR, Agarwal, RP, Pavani, R, Thandapani, E: On the oscillation of certain third order nonlinear functional differential equations. Appl. Math. Comput. 202(1), 102-112 (2008)

\section{Submit your manuscript to a SpringerOpen ${ }^{\circ}$ journal and benefit from:}

- Convenient online submission

- Rigorous peer review

Open access: articles freely available online

- High visibility within the field

- Retaining the copyright to your article

Submit your next manuscript at $\gg$ springeropen.com 